\title{
Low-Loss EELS Study of Oxide-Covered Aluminum Nanospheres
}

\author{
Thomas Stöckli $\left({ }^{1}\right)$, Pierre Stadelmann $\left({ }^{2}\right)$ and André Châtelain $\left({ }^{1}\right)$ \\ ( ${ }^{1}$ Institut de Physique Expérimentale, Département de Physique, \\ École Polytechnique Fédérale de Lausanne, 1015 Lausanne, Switzerland \\ $\left({ }^{2}\right)$ Centre Interdépartemental de Microscopie Électronique, \\ École Polytechnique Fédérale de Lausanne, 1015 Lausanne, Switzerland
}

(Received December 30, 1996; accepted May 15, 1997)

\author{
PACS.73.20 - Electronic surface states \\ PACS.71.45.Gm - Exchange, correlation, dielectric and magnetic functions, plasmons \\ PACS.79.60.-i - Photoemission and photoelectron spectra
}

\begin{abstract}
High resolution transmission electron microscopy (HRTEM) and electron energy loss spectroscopy (EELS) have been used to investigate plasmon losses of aluminum nanospheres. A model based on the dielectric theory allows to attribute the observed features in the loss spectra of particles of different size to either surface or volume losses. Both, surface and volume loss peak, show size dependent characteristics which are reproduced by this model. EEL spectra with negligible surface loss contributions can be used to determine the dielectric function of the observed material by means of the Kramers Kronig relations. For the case of non negligible surface contributions we introduce the notion of dielectric signature. This dielectric signature allows to study the transition where surface effects start to become visible in the spectra. Our analysis suggests that for a system of known composition this approach is a useful tool to determine the importance of surface effects.
\end{abstract}

\section{Introduction}

Electron energy loss spectroscopy (EELS) in a high resolution transmission electron microscope (HRTEM) is a well suited experimental tool for the investigation of the electronic properties on a nanometer scale [1]. This is particularly interesting in the case of small particles (size in the nanometer range), since due to surface effects, their electronic properties differ from those of the bulk material. EELS allows to investigate the electronic properties of different isolated particles, and thus to study the surface-induced changes of the electronic response of the particles.

The EEL spectra obtained from aluminum nanospheres of different size are analysed in two ways: the first approach presented in Section 3 consists in analysing the observed peaks in terms of a dielectric theory model [2-9]. If the geometry of the particle is simple, this model permits an attribution of the experimental peaks to either surface or volume plasmon excitations. It predicts furthermore a dependence of the resonance energy on the geometrical parameters of the particle. The geometrical parameters of the aluminum nanospheres being known from HRTEM observations, it is possible to test the predictions of the model. 


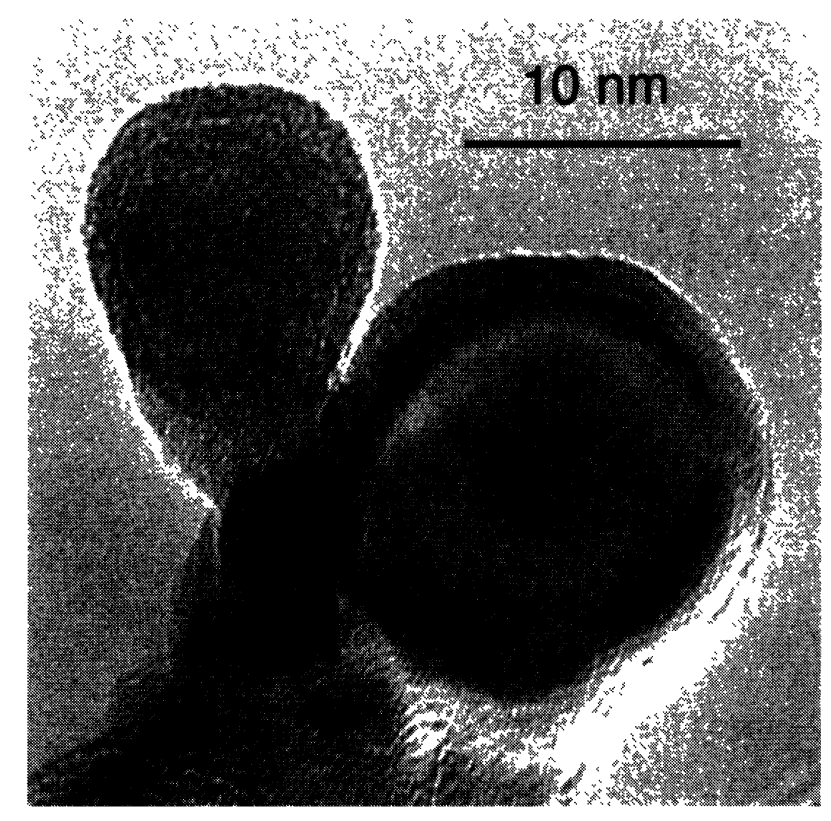

Fig. 1. - Bright field TEM micrograph of typical $\mathrm{Al}$ nanospheres. The oxide layer and the $\mathrm{Al}$ core can clearly be distinguished.

The second approach (Sect. 4) uses the property that the single scattering contribution due to the volume loss is proportional to the imaginary part of the inverse of the dielectric function $[1,10]$. If surface effects are negligible, this single scattering contribution can be obtained from the experimental spectra by a Fourier Log deconvolution $[1,11]$. The causality of the response of the system then allows to apply Kramers Kronig relations to determine the dielectric function $[1,12]$. Our measurements were however carried out on particles covering the range where surface effects begin to be observable and become important. The limit of validity of the determination of the dielectric function via Kramers Kronig relations in such a system is not well defined, since it is difficult to determine if surface losses are negligible or not. In order to better define this limit, we propose to introduce a mathematical object called dielectric signature. It can be obtained from the deconvoluted loss spectrum (containing surface effects) using Kramers Kronig relations. Its principal property is that it converges towards the dielectric function of the observed material if surface effects become negligible. We propose to use this dielectric signature to study EEL spectra with increasing surface loss contributions in order to find out when they become too important to be negligible.

\section{Experimental}

Al nanospheres were produced using the well known vapour deposition in a He atmosphere as described in detail by Yatsuya et al. [13]. Evaporation temperature, chamber pressure and the distance between source and sample holder determine the size of the particles. The spheres were directly evaporated on holey carbon film microgrids. No particular precautions were taken to prevent oxidation during transport to the microscope. Figure 1 shows typical Al nanospheres produced by this method. The oxide layer can easily be distinguished from the $\mathrm{Al}$ core. 


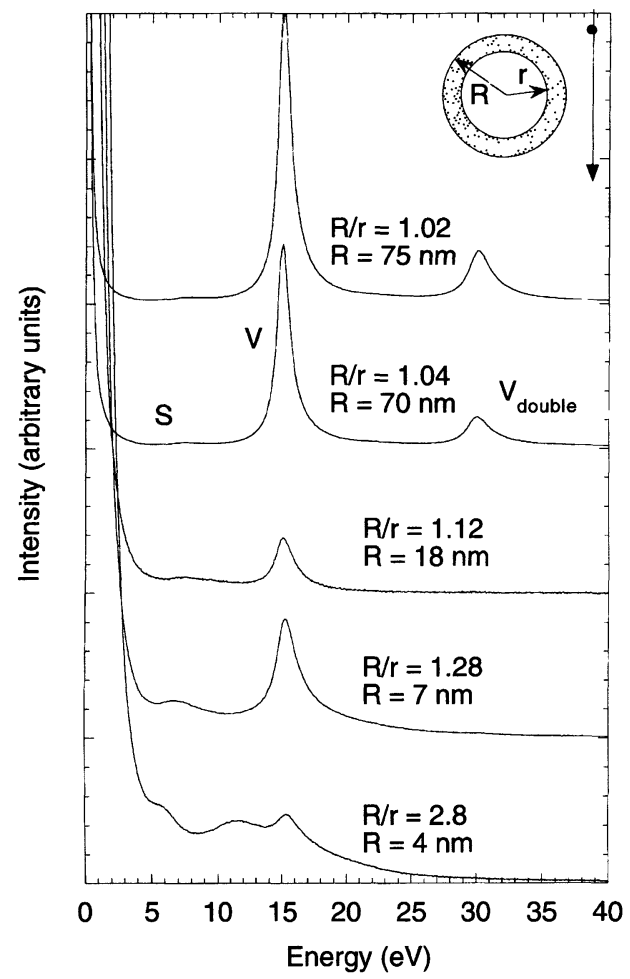

Fig. 2. - EEL spectra of $\mathrm{Al}$ nanospheres with different radius $R$ and ratio $R / r . R$ is the radius of the whole particle (Al core and the oxide-shell), and $r$ is the radius of $\mathrm{Al}$ core only. $V$ indicates the volume plasmon loss, $V_{\text {double }}$ the corresponding double loss and $S$ the surface plasmon loss.

The microscope used for the investigation was a Hitachi HF 2000 equipped with a field emission gun operated with an acceleration voltage of $200 \mathrm{kV}$. The samples were cooled to liquid nitrogen temperature to prevent beam-induced contamination. For spectrum acquisition, the beam was focused to a probe of a diameter of $2 \mathrm{~nm}$ and placed on the centre of the particle. The loss spectra were recorded with a Gatan 666 parallel detection EEL spectrometer with an energy resolution of $0.5 \mathrm{eV}$ (FWHM of the zero loss peak) and a maximum dispersion of $0.05 \mathrm{eV}$ per channel. Measurements were carried out on particles on the edge of the holes in the carbon film in order to avoid the amorphous carbon background.

\section{Interpretation of the Spectra by Means of the Dielectric Theory}

Figure 2 shows EEL spectra for different $\mathrm{Al}$ nanospheres. For the diameter range covered by the investigated particles $(10$ to $100 \mathrm{~nm})$ it can be expected that surface effects are non negligible and that the spectra contain surface loss contributions. In order to assign the different observed peaks to either volume or surface losses, simulations based on dielectric theory have been carried out.

For the volume plasmon it is well established that the observed intensity $J^{1}(E)$ is proportional to the loss function $\operatorname{Im}(-1 / \varepsilon(E))$, where $\varepsilon(E)$ is the dielectric function of the investigated 

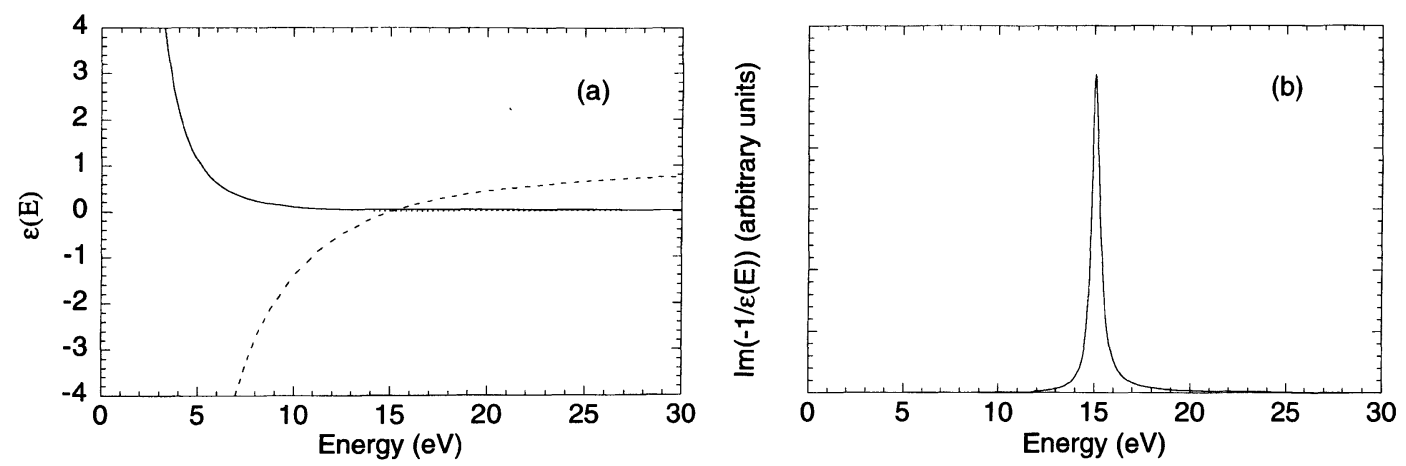

Fig. 3. - a) Real and imaginary part of the dielectric function of aluminum determined by optical measurements (from Ref. [14]). The real part is shown by the dotted line and the imaginary part by the solid line. b) Loss function calculated from the dielectric function of aluminum.

medium $[1,10]$ :

$$
J^{1}(E)=\frac{2 I_{0} t}{\pi a_{0} m_{0} v^{2}} \operatorname{Im}\left(-\frac{1}{\varepsilon(E)}\right) \ln \left(1+\left(\frac{\beta}{\theta_{E}}\right)^{2}\right) \propto \operatorname{Im}\left(-\frac{1}{\varepsilon(E)}\right) .
$$

The factor preceding $\operatorname{Im}(-1 / \varepsilon(E))$ contains the beam intensity $\left(I_{0}\right)$, the velocity of the incoming electrons $(v)$, the sample thickness $(t)$ and as fundamental constants the Bohr radius $\left(a_{0}\right)$ and the rest mass of the free electron $\left(m_{0}\right)$. The term in the logarithm contains the collection semiangle of the detector $(\beta)$ and the characteristic scattering angle for an energy loss of energy $E\left(\theta_{E}\right)$.

Using the dielectric function of aluminum [14] (see Fig. 3a), the loss function shown in Figure $3 \mathrm{~b}$ can be calculated. By comparison with the observed peaks, we can attribute the energy loss peak at $15 \mathrm{eV}$ to the $\mathrm{Al}$ volume plasmon $(V)[1,15]$. If the sample is thick enough (i.e. if the trajectory of the particle is longer than the mean free path of the electron), the incoming electron can excite more than one plasmon. The corresponding double loss peak $\left(V_{\text {double }}\right)$ at $30 \mathrm{eV}$ is therefore only observed for the largest particles. In Figure 4, measured characteristics of the volume plasmon loss are reported as a function of the radius $r$ of the $\mathrm{Al}$ core of the particle (oxide layer not included). Figure 4a shows that the measured resonance energies are in the range from 15 to $15.1 \mathrm{eV}$ if the core radius is larger than $5 \mathrm{~nm}$. These values are in good agreement with those found in literature $[1,15,16]$. For Al core radii smaller than $5 \mathrm{~nm}$, the resonance energy shifts to higher values. Such a shift of the volume plasmon resonance energy has also been reported for oxidized and non oxidized $\mathrm{Ga}$ and $\mathrm{Sn}$ spheres $[17,18]$. In that case, it has been shown that the blue shift is due to dispersion effects in a finite medium. Detailed theoretical analysis on supported oxide covered particles using a hydrodynamic model [8] show that dispersion effects can in fact be expected for particles with metal core radii smaller than $4 \mathrm{~nm}$. On the other hand it has to be noted, that for the smallest particles there is an additional absorption feature at $11.5 \mathrm{eV}$ appearing (see below). The experimentally observed blue shift is therefore most likely due to the combined effects of dispersion in a finite medium and the superposition of the absorption at $11.5 \mathrm{eV}$.

Figure $4 \mathrm{~b}$ shows the measured full width at half maximum (FWHM) of the volume plasmon loss peak. Its value increases with decreasing particle size. The dotted line corresponds to a fit of a $r^{-1}$ type curve as predicted by the Drude theory [19]: FWHM $=\gamma=\gamma_{\text {bulk }}+k\left(v_{\mathrm{F}} / r\right)$ 

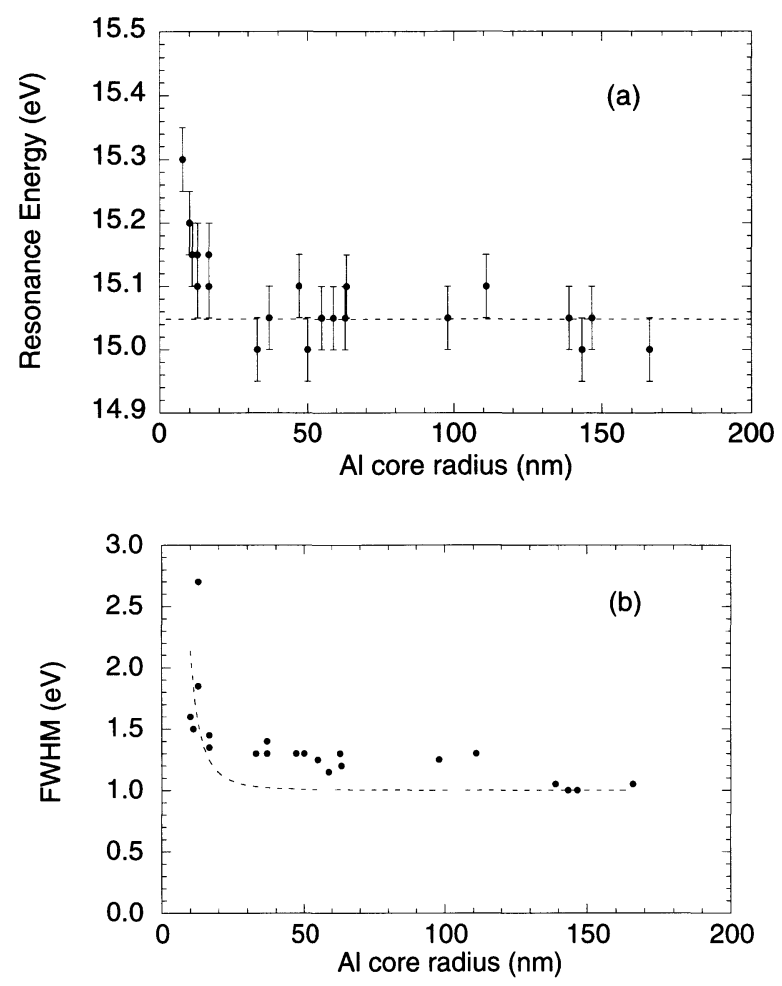

Fig. 4. - a) Resonance energy of $\mathrm{Al}$ volume plasmon and b) full width at half maximum of the volume plasmon peak as a function of the $\mathrm{Al}$ core radius $r$. The dashed line corresponds to a fit of a $r^{-1}$ type curve (see text).

where $\gamma$ is the metal damping constant, $\gamma_{\mathrm{bulk}}$ is the bulk damping constant, $v_{\mathrm{F}}$ is the electron velocity at the Fermi surface and $k$ a constant.

The volume loss being identified, it is necessary to test whether the feature appearing between 6 and $8 \mathrm{eV}$ (depending on the geometrical parameters of the particles) corresponds to the surface losses. For this purpose a dielectric theory model taking into account the effect of the oxide layer $[1,20-22]$ on the spheres (typically 3 to $4 \mathrm{~nm}$ thick) has been used to calculate resonance energy and excitation probability of the surface loss.

In this model, several hypothesis are made. In particular, three effects are neglected: the quantum size effect, the spatial dispersion effect and the retardation effect. When the size of the particle becomes very small, quantum-mechanical effects (inducing a shift of the resonance frequencies towards higher energies) can occur. It is however known that these effects are negligible for particles with radii greater than a couple of nanometers. For the spatial dispersion, Wang and Cowley [8] have demonstrated that its effects are negligible for particles with diameters greater than $8 \mathrm{~nm}$. Finally Boardman and Paranjape [23] have calculated the influence of retardation effects and shown that they can be neglected for particles smaller than $20 \mathrm{~nm}$. Since the diameters of the observed particles are in the range between 10 and $300 \mathrm{~nm}$, we conclude that the model should be adequate to explain most of our experimental data.

If $R$ is the radius of the entire particle ( $\mathrm{Al}$ core and oxide layer) and $r$ is the radius of the $\mathrm{Al}$ core as before (see inset Fig. 2), the dielectric theory model predicts a dependence of the surface plasmon resonance energy on the ratio $R / r$. The surface plasmon resonance frequencies 


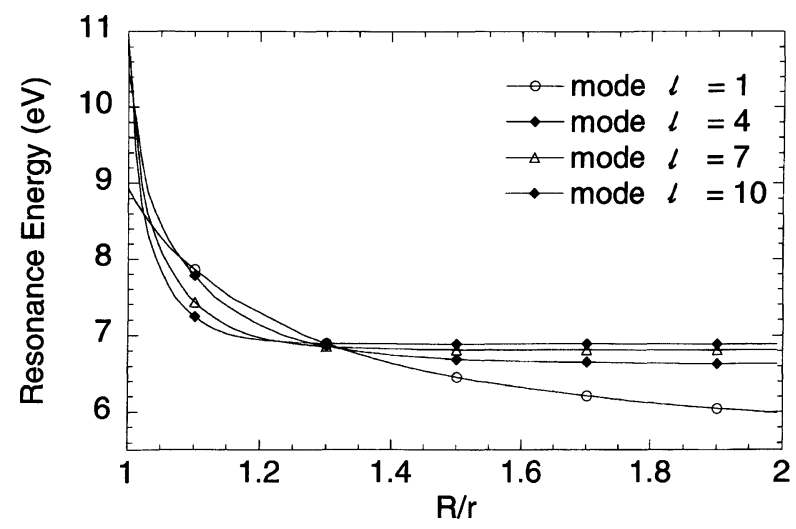

Fig. 5. - Calculated resonance frequencies for different modes of oscillation of the oxide covered aluminum sphere as a function of the parameter $R / r$.

$\omega_{\mathrm{s}}$ are determined for a given ratio $R / r$ by the relation [22]:

$$
\begin{aligned}
\varepsilon_{2}^{2}\left(\omega_{\mathrm{s}}\right) & +\frac{\varepsilon_{2}\left(\omega_{\mathrm{s}}\right)}{1-(R / r)^{2 \ell+1}}\left[\varepsilon_{0}\left(\omega_{\mathrm{s}}\right)\left(\frac{\ell+1}{\ell}+\left(\frac{R}{r}\right)^{2 \ell+1}\right)+\varepsilon_{1}\left(\omega_{\mathrm{s}}\right) \frac{\ell}{\ell+1}+\left(\frac{R}{r}\right)^{2 \ell+1}\right] \\
& +\varepsilon_{1}\left(\omega_{\mathrm{s}}\right) \varepsilon_{0}\left(\omega_{\mathrm{s}}\right)=0 .
\end{aligned}
$$

$\varepsilon_{0}(\omega), \varepsilon_{1}(\omega)$ and $\varepsilon_{2}(\omega)$ are the frequency dependent dielectric functions of empty space, the aluminum core and the oxide layer respectively. $\ell$ stands for the mode of oscillation and can take the values $\ell=1,2,3, \ldots$ The dependence of the resonance frequency $\omega_{\text {s }}$ on the ratio $R / r$ is shown in Figure 5 for different values of $\ell$. In order to determine the dominant mode, it is necessary to calculate the excitation probability of the surface plasmon. If one is only interested in the position of the surface peak (and not in its intensity compared to the volume peak), the excitation probability can be calculated for an electron passing just outside the particle. For the computation of the excitation probabilities, the results of Ferrell et al. [22] (see Appendix) have been used. In Figure 6, partial sums over the excitation modes $\ell$ are shown. It can be seen that the contributions of the modes $\ell \geq 2$ don't significantly change the position of the peak. It is therefore justified to compare the measured surface plasmon resonance energies with the theoretical values for the mode $\ell=1$. Figure 7 shows a comparison between the measured values and the theoretical predictions for the mode $\ell=1$. It can be seen that the general tendency of the measured values follows the theoretical values. Since the thickness of the oxide layer was always about $4 \mathrm{~nm}$, the dependence of the resonance energy on the ratio $R / r$ could be measured only by varying the particle size. This means that for values or $R / r$ close to 1 the particles become rather large and inversely they become rather small for large values of $R / r$. Typically, the diameter of the particle for the value of $R / r$ closest to 1 was $300 \mathrm{~nm}$ and, the one for the highest measured value of $R / r$ was $15 \mathrm{~nm}$. These values are close to the theoretical limits of the model, especially for $R / r$ close to 1 . This might explain why the measured resonance energy for the smallest values of $R / r$ are found to be low compared to the model.

In the case of the smallest observed particles, an additional absorption can be observed at $11.5 \mathrm{eV}$ (see Fig. 2). The model treating an oxide covered sphere in empty space like it was used above does not account for this feature. It has to be noted, that in that case, the effect of the support on the plasmon resonances has been neglected. In a more detailed calculation taking 

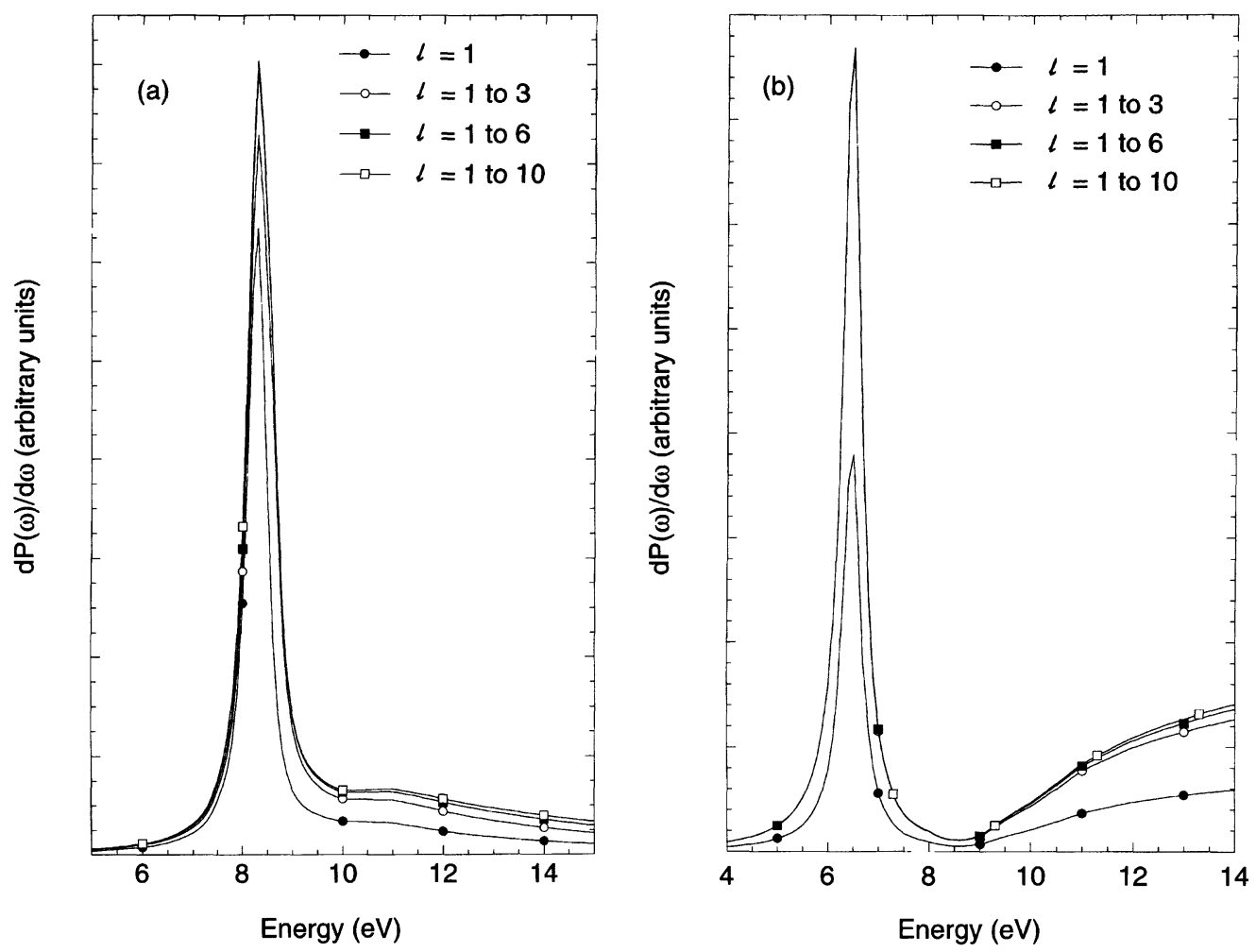

Fig. 6. - Simulated excitation probabilities of the surface plasmon for a ratio $R / r$ of 1.05 (a) and a ratio $R / r$ of 1.5 (b). It can be seen that the mode $\ell=1$ is dominating for both values of $R / r$. The contributions of higher order modes do not shift the peak position.

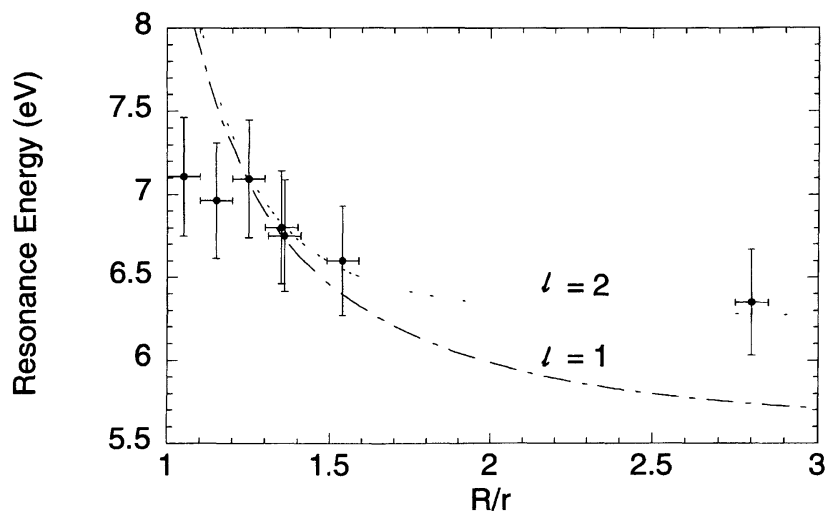

Fig. 7. - Experimental and theoretical (dielectric theory simulation) values of the surface plasmon resonance energy plotted versus the ratio $R / r$. 
into account the effect of the support, Wang et al. [6-8] have shown that resonance modes other than the one appearing in the oxidized sphere (without support) can exist. In particular, resonances at 10 and at $11.5 \mathrm{eV}$ for an oxidized $\mathrm{Al}$ sphere on a conducting support are found. These losses are attributed to the coupling excitation of the $\mathrm{Al}_{2} \mathrm{O}_{3}$ shell and the support. In their calculations, the support was made of $\mathrm{Al}$, not of graphite as in our measurements. The particle-support coupling modes responsible for the absorption at energies between the surface excitation and the volume excitation however also exist for other conducting supports. We therefore attribute the feature appearing at $11.5 \mathrm{eV}$ in the spectra of the smallest particles to the coupling excitation between the oxide covered particle and the support.

\section{Interpretation of the Spectra Using Kramers Kronig Relations}

As indicated in the previous section, the energy loss of small particles contains two contributions: a first term due to the volume losses and a second term due to the surface losses.

$$
I_{\text {total }}(E) \propto I_{\text {volume }}(E)+I_{\text {surface }}(E) .
$$

If the sample is thick enough, the surface contribution is negligible compared to the volume contribution and it is possible to extract the single scattering distribution via a Fourier Log deconvolution $[1,11]$. The single scattering contribution is proportional to the imaginary part of the inverse of the dielectric function of the bulk material (see Eq. (1)). Using Kramers Kronig relations, it is then possible to deduce the complex dielectric function of the bulk material. In Figure 8, the dielectric function of bulk aluminum measured on an Al film is plotted (solid line). In this case, EELS measurements have been carried out on an Al film of sufficient thickness $(150 \mathrm{~nm})$ to neglect surface effects, and therefore the free electron behaviour is observed. A comparison between the dielectric function determined by EELS and the one determined by optical measurements (Fig. 3) demonstrates the validity of this approach if surface effects are negligible. However, surface effects are visible in the EEL spectra of the aluminum nanospheres (see Sect. 3). Naturally, surface effects become less important as the ratio volume over surface increases. In order to better define at what moment they become negligible, we have applied the Kramers Kronig approach to EEL spectra with increasing surface contribution. From a mathematical point of view this means that the total energy loss containing surface and volume contribution given in equation (3) is associated to the imaginary part of a purely mathematical object that we propose to call the dielectric signature of the particle $\varepsilon_{\mathrm{s}}(E)$ :

$$
I_{\text {total }}(E) \propto I_{\text {volume }}(E)+I_{\text {surface }}(E) \propto \operatorname{Im}\left(-\frac{1}{\varepsilon_{\mathrm{s}}(E)}\right) .
$$

$I_{\text {total }}(E)$ is the measured intensity of the EEL spectrum and represents the physical response of the system (aluminum nanospheres) to the incoming electrons. The causality of the system is therefore assured and Kramers Kronig relations can be used to determine the real part of $1 / \varepsilon_{\mathrm{s}}(E)[24]$. Knowing the real and the imaginary part of the inverse of the dielectric signature $\varepsilon_{\mathrm{s}}(E)$, it is possible to deduce the complex dielectric signature. Figure 8 shows the resulting dielectric signature of the experimental spectra shown in Figure 2. The dielectric signature is different from the dielectric function of aluminum (solid line): the imaginary part of the dielectric signature (Fig. 8a) shows a peak which cannot be seen in the dielectric function of aluminum. Its height increases and the position of the peak maximum is shifted to higher energies as surface effects become more important. The same tendency can be observed for the real part of the dielectric signature (Fig. 8b). It is important to notice that surface losses hardly visible in the original EEL spectrum (Fig. 2) appear clearly as peaks in the calculated 

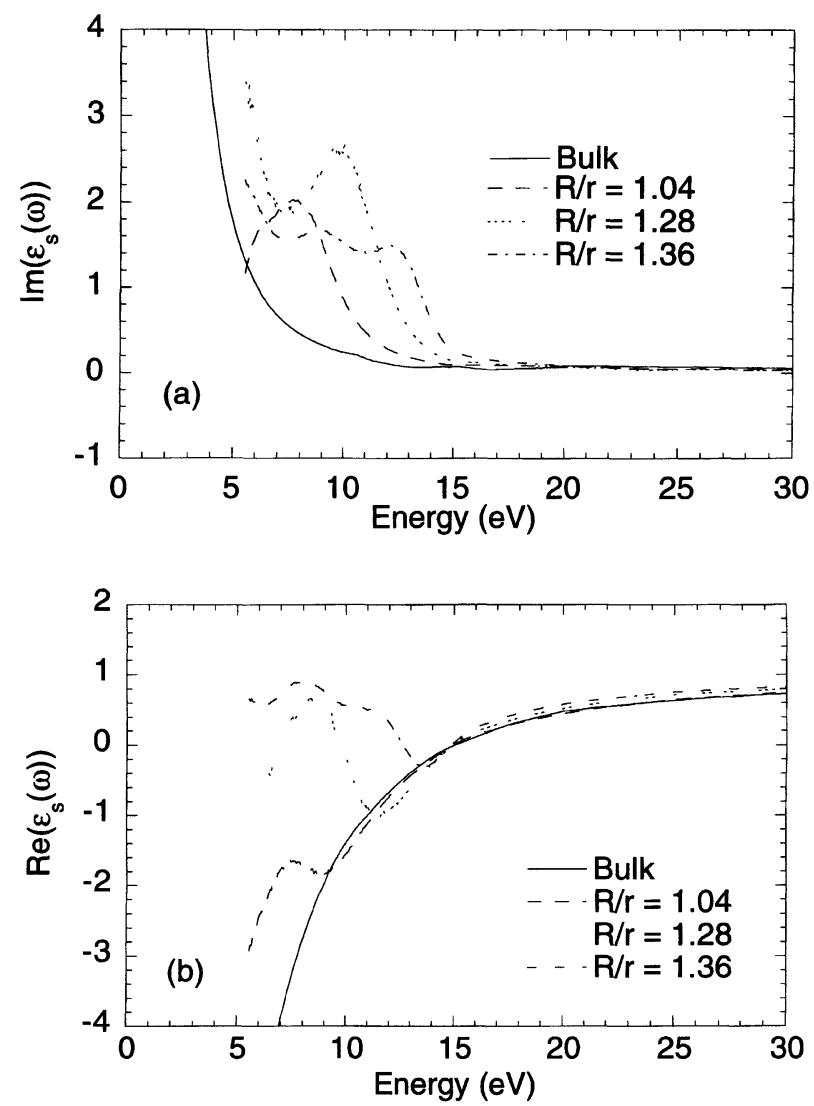

Fig. 8. - Imaginary (a) and real (b) part of the dielectric signature calculated via Kramers Kronig relations. If surface effects are negligible, the dielectric signature is identical to the dielectric function of aluminum. If surface effects are present, additional peaks appear.

dielectric signature. For a system of known composition, this tendency can therefore be used to determine more accurately, if surface effects are present in the EEL spectra or not.

\section{Conclusion}

Two possible ways of analysing low loss EEL spectra are tested on oxide covered aluminum spheres. In a first part, we use a model based on dielectric theory to associate the observed features in the experimental spectra to either volume or surface losses. Both, volume and surface losses show size dependent characteristics. The variations of the FWHM of the volume loss peak follow the predictions of the Drude model. In the case of the surface losses, experimental values compare well to predictions of the dielectric theory, even close to the limit of validity of the model. Our results show that the dielectric theory describes successfully the plasmon losses in small spheres.

In a second part, we try to determine at what particle size surface effects become negligible. This is important to know since the determination of the dielectric function of the particle via Kramers Kronig relations is only possible if surface effects are negligible. Generally, it is not 
easy to determine if this is true. In the purpose to better define the limit, we introduce the dielectric signature. This dielectric signature is shown to be a useful tool to determine the importance of surface losses in a EEL spectra, if the dielectric function of the system is known.

\section{Acknowledgments}

The authors would like to thank Z.L. Wang for his careful reading of the manuscript and important comments. They would also like to thank Ch. Colliex, W. de Heer, D. Ugarte and J.M. Bonard for many helpful discussions. They would like to acknowledge M. Fazan and G. Peter for technical support. This work was partially funded by the Swiss National Science Foundation.

\section{Appendix}

The following formulae describe the excitation probability for an oxide covered sphere in the case of an electron passing just outside the sphere. The surface excitation probability is given by the sum over the excitation probabilities of the different modes:

$$
P_{\text {surface }}(d, \omega)=\sum_{\ell \geq 1} P_{\ell}(d, \omega)
$$

where $d$ is the impact parameter $(d \geq R)$ and $P_{\ell}(d, \omega)$ is given by:

$$
P_{\ell}(d, \omega)=\frac{e^{2} R}{\pi^{2} \varepsilon_{0} \hbar^{2} v^{2}} \sum_{m=0}^{\ell} \frac{2-\delta_{m, 0}}{(\ell-m) !(\ell+m) !} \operatorname{Im}\left(-\gamma_{\ell}(\omega)\right)\left(\frac{\omega R}{v}\right)^{2 \ell} K_{m}^{2}\left(\frac{\omega d}{v}\right) .
$$

$K_{m}$ is the $\mathrm{K}$ type Bessel function of order $m, \delta_{m, 0}$ the Kronecker symbol, $e$ the elementary charge and $v$ the speed of the incoming electron. $R$ and $d$ are defined as before, e.g. $R$ is the particle radius and $d$ the impact parameter. The dielectric functions of the different media $\left(\varepsilon_{0}(\omega)\right.$ for empty space, $\varepsilon_{1}(\omega)$ for the aluminum core and $\varepsilon_{2}(\omega)$ for the oxide layer) are contained in the expression $\gamma_{\ell}(\omega)$ :

$$
\gamma_{\ell}(\omega)=\frac{\ell\left(1-\varepsilon_{2}(\omega)\right)+f_{\ell}\left[\ell+(\ell+1) \varepsilon_{2}(\omega)\right](R / r)^{-(2 \ell+1)}}{\ell \varepsilon_{2}(\omega)+(\ell+1)+f_{\ell}(\ell+1)\left(1-\varepsilon_{2}(\omega)\right)(R / r)^{-(2 \ell+1)}}
$$

with

$$
f_{\ell}=\frac{\ell\left(\varepsilon_{2}(\omega)-\varepsilon_{1}(\omega)\right)}{\ell \varepsilon_{1}(\omega)+(\ell+1) \varepsilon_{2}(\omega)}
$$

\section{References}

[1] Egerton R.F., Electron Energy Loss Spectroscopy in the Electron Microscope (Plenum Press, New York and London, 1989).

[2] Echenique M., Howie A. and Wheatley D.J., Philos. Mag. B 56 (1987) 335-345.

[3] Ferrell T.L. and Echenique P.M., Phys. Rev. Lett. 55 (1985) 1562-1529. 
[4] Ferrell T.L., Anderson V.E., Echenique P.M. and Warmack R.J., Phys. Rev. B 35 (1987) 7365-7317.

[5] Ugarte D., Colliex C. and Trebbia P., Phys. Rev. B 45 (1992) 4332-4343.

[6] Wang Z.L. and Cowley J.M., Ultramicroscopy 21 (1987) 77-93.

[7] Wang Z.L. and Cowley J.M., Ultramicroscopy 21 (1987) 335-346.

[8] Wang Z.L. and Cowley J.M., Ultramicroscopy 23 (1987) 97-107.

[9] Zabala N. and Rivacoba A., Ultramicroscopy 35 (1991) 145-150.

[10] Ritchie R.H., Phys. Rev. 106 (1957) 874-881.

[11] Johnson D.W. and Spence J.C.H., J. Phys. D 7 (1974) 771-780.

[12] Johnson D.W., J. Phys. A 8 (1974) 490-495.

[13] Yatsuya S., Kasukabe S. and Uyeda R., Jpn J. Appl. Phys. 12 (1973) 1675-1684.

[14] Palik E. D., Handbook of Optical Constants of Solids I and II (Academic Press Inc., New York, 1985).

[15] Raether H., Pick H. and Bennemann K. H., Solid State Excitations by Electrons (Springer Verlag, Berlin, Heidelberg New York, 1965).

[16] Daniels J., Festenberg D. and Raether H., Optical Constants of Solids by Electron Spectroscopy (Springer Verlag, Berlin, Heidelberg and New York, 1979).

[17] Achèche M., Colliex C., Kohl H., Nourtier A. and Trebbia P., Ultramicroscopy 20 (1986) 99-106.

[18] Achèche M., Colliex C. and Trebbia P., Scanning Electron Microscopy (1986) 25-32.

[19] Bohren C.F. and Huffman D.R., Absorption and Scattering of Light by Small Particles (John Wiley \& Sons, New York, 1983).

[20] Ugarte D., Ph D Thesis, Université de Paris-Sud, Centre d'Orsay (1990).

[21] Ugarte D., Colliex C. and Trebbia P., Phys. Rev. B 45 (1992) 4332-4343.

[22] Ferrell T.L., Warmack R.J., Anderson V.E. and Echenique P.M., Phys. Rev. B 35 (1987) 7365-7371.

[23] Boardman, A.D. and Paranjape B.D., J. Phys. F 7(1977) 1935-1941.

[24] Jackson J.D., Classical Electrodynamics (John Wiley \& Sons, New York, 1975) 